\title{
Electron transfer theory and its inception
}

\author{
Rudolph A. Marcus \\ DOI: $10.1039 / \mathrm{c2cp90116a}$
}

It is a pleasure to introduce this issue on electron transfer processes. The field has developed greatly and in many different directions not envisioned in the late 1940s. The modern electron transfer era began at that time in the form of studies on the simplest class of reactions in all of chemistry, isotopic exchange reactions of the electron transfer type. In their simplest form no chemical bonds are broken or formed, only an electron is transferred from one reactant to the other. I remember how surprised and excited I was reading in 1955 a paper by Bill Libby (W. F. Libby, J. Phys. Chem., 1952, 56, 893), written several years earlier, explaining why some of these reactions were slow and others fast. In his explanation he used the Franck-Condon principle to interpret the results: he noted that when an electron "jumped" from one reactant to the other the slow moving nuclei changed neither their positions nor their momenta during the jump, and what the consequences were. I was especially excited, since that principle had originally been introduced to explain molecular spectra rather than chemical reaction rates. But perhaps at this point I should say a few words on how I came into theoretical chemistry as a practitioner just a few years earlier.

As a student at McGill University in Montreal, where I was born, the research was entirely experimental. There were no theoretical chemists in Canada at that time, and so all of us chemists were perforce experimentalists. My $\mathrm{PhD}$ research was on rates of chemical reactions in solution, directed by Carl Winkler, a Canadian who had in turn received his $\mathrm{PhD}$ under the guidance of the later

Noyes Laboratory of Chemical Physics, California Institute of Technology, Pasadena, CA 91125
Nobel Laureate Cyril Hinshelwood at Oxford in the 1930s. When I was a student only McGill and Toronto Universities awarded PhD's in Canada in chemistry, the remaining universities limiting their graduate offerings to MSc's. In physical chemistry McGill's strength was in kinetics and Toronto's was thermodynamics. All of this was well known to the graduate students. After completing my PhD in 1946 I went to the National Research Council of Canada in Ottawa to do postdoctoral experimental research, again in chemical kinetics, but now on gas phase photochemical rates with B. de B. Darwent and with Canada's renowned E. W. R. Steacie. My second paper was published with Steacie in 1949 as part of the Bonhoeffer Festschrift, Steacie having worked with Bonhoeffer in Germany in the 1930s.

At that point after some five years of experiments I began to feel uneasy, coming to realize that my love for math was much more suited to theory. I gambled on trying a second postdoctoral, this time in theory, and with Oscar K. Rice, a marvelous theoretician at the University of North Carolina. More likely, it was Rice who gambled, since I had zero prior research experience in theory.

The move to theory proved to be a dramatic experience, unexpectedly fruitful as well as delightful. I loved the experience and the " $24 / 7$ " learning. The problem that I settled on after three months reading and sitting in on some classes developed into what later become known as RRKM theory (RiceRamsperger-Kassel-Marcus) of unimolecular reactions and bimolecular recombinations $(1951,1952)$. It was initiated at Carolina and completed at the Polytechnic Institute of Brooklyn, my first faculty position. It also continues be the standard theory in its field.
At Brooklyn Poly I began directing experimental research with several graduate students, and wondered what to do for theoretical studies. In a sense I was on my own in theory, with only one theory graduate student during my 13 years there but quite a few experimental students. I decided not to continue with unimolecular reaction rate theory, for which there were no new experimental data. Indeed, one of our experimental projects was to obtain such data. In retrospect, not continuing the unimolecular theory study, a barren prospect at the time, was a fortunate decision. Time spent on it would have reduced the time available for working on new projects, if they arose, another type of gamble. Fortunately, my experimental work with the research group meant that the research itself was not at a standstill, only the theoretical part!

I worked on a few small theoretical problems, including the statistics of isotopic exchange in gas phase boron hydride reactions, but received my first "break" when a student in a statistical mechanics course I was teaching asked me a question about a polyelectrolyte problem that he himself was studying in the laboratory. In exploring an answer I looked at every book specializing in electrostatics available in the Brooklyn Poly library, some 11 in all, and found one (Mason and Weaver) that seemed more fundamental in its treatment. It went substantially beyond the course I had taken as a sophomore at McGill in electricity and magnetism. I studied it and wrote a couple of papers on electrostatic properties and free energy of polyelectrolytes $(1954,1955)$. When the electron transfer problem came along shortly afterwards I was, with this detailed electrostatics background, "ready" for it, and in one month derived the electron 
transfer equation for the free energy barrier and the rate constant.

With this digression on background I return to Libby's electron transfer paper. After the initial excitement on reading it, I became uneasy and realized that something was amiss. Although the FranckCondon part was correct, namely Libby's idea on when there would be a large change in energy before and after the electron transfer, reflecting changes in equilibrium bond lengths and equilibrium solvation of each reactant, and hence a large energy barrier to the transfer, the approach I realized led to a violation of the law of energy conservation. Where was the extra energy to come from? What had to occur prior to the transfer was a reorganization of the environment to permit the electron jump to occur with no change in energy. After the electron transfer the environment could relax further to its new equilibrium configurations. After that it was a matter of some analysis using an expression I obtained for the free energy of nonequilibrium fluctuations of the environment (nonequilibrium dielectric polarization), fluctuations that permitted the electron transfer. I had read and was no doubt influenced by the physics literature on polaron theory, a very active field in physics at the time. Pekar's classic book on the polaron was translated from the Russian to German, but after that one was on one's own!

Theory then was, of course, quite different from now. Analytical theory, including the art of constructing physically based approximations, is still important, but as a percentage of total theoretical research effort it is now small compared with computation. I believe that the future can be expected to settle on some insightful combination of both, since both are necessary.

For interaction of experimentalists and theoreticians the application of analytical expressions can be especially attractive when they are relatively simple. I recall examples from my own work, one being the symmetrization of potential energy surfaces of reactants and products to simplify the electron transfer theory, and its amplification and correction relegated to an Appendix of a 1965 paper. That correction is now finding use, some 45 years later, in interpreting small deviations from electrochemical Tafel slopes of 0.5 in the vicinity of zero overpotential. Complicated looking expressions may discourage even the most ardent experimentalist supporter of analytical theory, unless the stakes are unusually high!

When I worked on electron transfers it was purely trying to solve an interesting problem, with no thought that there might be major consequences and no grand plan. It happened later that the field turned into an extremely broad and important one, because of the widespread occurrence of electron transfers and the many applications.

Perhaps I should add that although my subsequent research drifted into many other areas, electron transfers stayed to be constantly the background in studies of mechanistic details and their experimental realization, a prominent one involving the primary processes of transmembrane electron translocation in photosynthesis. Recently, the treatment of electron transfer in ensembles has been complemented by single molecule studies that span a broad field. It includes charge carrier injection into semiconductor nanoparticles as components of solar energy converters and the intermittent fluorescence phenomena in semiconductor quantum dots. In these studies we have found that a diffusion controlled electron transfer theory provides an interpretation of the results as well as making predictions.

The current support of the author's research and that of his group by ONR, NSF and ARO is gratefully acknowledged. My early electron transfer work was supported by ONR and shortly thereafter also by NSF. 